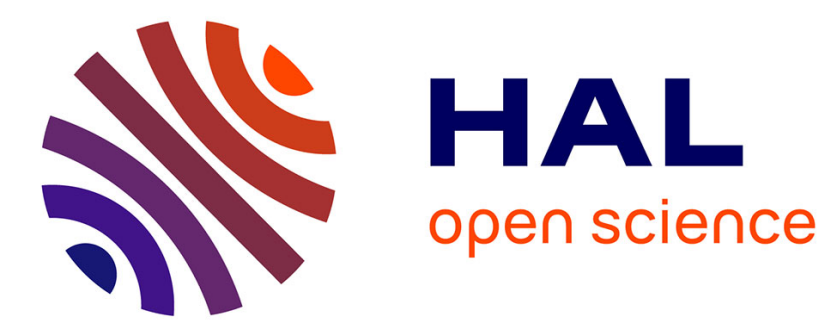

\title{
Our (represented) World: A Quantum-Like Object
}

Ariane Lambert-Mogiliansky, François Dubois

\section{To cite this version:}

Ariane Lambert-Mogiliansky, François Dubois. Our (represented) World: A Quantum-Like Object. 2015. halshs-01152332

\author{
HAL Id: halshs-01152332 \\ https://shs.hal.science/halshs-01152332 \\ Preprint submitted on 15 May 2015
}

HAL is a multi-disciplinary open access archive for the deposit and dissemination of scientific research documents, whether they are published or not. The documents may come from teaching and research institutions in France or abroad, or from public or private research centers.
L'archive ouverte pluridisciplinaire HAL, est destinée au dépôt et à la diffusion de documents scientifiques de niveau recherche, publiés ou non, émanant des établissements d'enseignement et de recherche français ou étrangers, des laboratoires publics ou privés. 


\section{PARISSCHOOLOFECQNOMICS}

WORKING PAPER N 2015 - 13

Our (represented) World: A Quantum-Like Object

Ariane Lambert-Mogiliansky

François Dubois

JEL Codes:

Keywords: Learning, Bohr complementarity, Information, Uncertainty

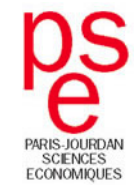




\title{
Our (represented) World: A Quantum-Like Object
}

\author{
Ariane Lambert-Mogiliansky ${ }^{a}$ and François Dubois ${ }^{b c}$ \\ ${ }^{a}$ Paris School of Economics, 48 Bd Jourdan, 75014 Paris, France. \\ ${ }^{b}$ Department of Mathematics, University Paris Sud, \\ Bât. 425, F-91405 Orsay Cedex, France. \\ ${ }^{c}$ Conservatoire National des Arts et Métiers, Paris, France, \\ Structural Mechanics and Coupled Systems Laboratory. \\ alambert@pse.ens.fr, francois.dubois@math.u-psud.fr.
}

30 April 20151

\begin{abstract}
It has been suggested that observed cognitive limitations may be an expression of the quantum-like structure of the mind. In this paper we explore some implications of this hypothesis for learning i.e., for the construction of a representation of the world. For a quantum-like individual, there exists a multiplicity of mentally incompatible (Bohr complementary) but equally valid and complete representations (mental pictures) of the world. The process of learning i.e., of constructing a representation involves two kinds of operations on the mental picture. The acquisition of new data which is modelled as a preparation procedure and the processing of data which is modelled as an introspective measurement operation. This process is shown not converge to a single mental picture but can evolve forever. We define a concept of entropy to capture relative intrinsic uncertainty. The analysis suggests a new perspective on learning. First, it implies that we must turn to double objectification as in Quantum Mechanics: the cognitive process is the primary object of learning. Second, it suggests that a representation of the world arises as the result of creative interplay between the mind and the environment. There is a degree of freedom that modifies the objective of rational learning.
\end{abstract}

Keywords: learning, Bohr complementarity, information, uncertainty.

AMS classification: 81Q99, 91C99.

\section{Introduction}

It is a common place that human beings are not capable of holding very complex picture in mind (cf "small worlds" in Savage 1954). We consider reality focusing on one perspective at a time and show difficulties in combining perspectives as amply documented. This inability to seize reality in its full richness suggests that the process of developing an understanding of the world may not look like a puzzle that is assembled

1 This contribution was presented at the Purdue Winer Memorial Lectures, 1-3 November 2014. To appear in Advanced Series on Mathematical Psychology, edited by Hans Colonius and Ehtibar Dzhafarov, World Scientific Review Volume. 


\section{Ariane Lambert-Mogiliansky and François Dubois}

progressively. Instead, the human mind may exhibit structural limitations in terms of the incompatibility of perspectives in a way similar to properties in quantum mechanics. Ambiguous pictures of the kind provided in diag. 1 provides a suggestive illustration of this phenomenon.

Quantum Mechanics is a theory about the physical world. Its object is sub-atomic particles. The well-known "strange" features arise when an observer attempts to learn about a sub-atomic system by means of a measurement device. A fundamental concept in QM is Bohr complementarity: two properties can be incompatible in the sense that they cannot have a determinate value simultaneously. As a consequence it is impossible to experimentally access that physical world without interacting with it in a non-deterministic manner. Intrinsic indeterminacy fundamentally changes the way we have to think about the physical reality or at least about the physical reality that we can have some knowledge about. It introduce non-separability between the measurement process and the world. There is no (accessible) given "outside world" but a contextual world in which we play an active role.

For some time the quantum paradigm has been proposed to described among other things aspects of the human psyche including preferences, attitudes and judgements in decision theory and psychology (Lambert-Mogiliansky 2009, Busemeyer Bruza 2012, Khrenikov 2010 among others). In this paper we take a step forward in recognizing that knowledge is also an (psychic) object which in itself may exhibit non-classical features and we derive some implications of this hypothesis for learning.

The approach can be viewed as a reversal of standard QM in the following sense. A person considers a classical object e.g., a state agency, but her knowledge about it i.e., the mental picture in her mind can behave in a non-classical way. Since human beings makes decisions on the basis of perceived reality i.e., of mental pictures, our approach extends the possible practical relevance of the quantum paradigm beyond sub-atomic physics to much of macroscopic reality.

The most important element of our theory is Bohr-complementarity of mental perspectives of one and the same object. They parallel that of properties for sub-atomic particles e.g., spin along different angles. We propose that Bohr complementarity of perspectives captures the cognitive limitations that are responsible for our difficulties to synthese information along different perspectives into one single coherent picture. The difficulties arise from the impossibility for incompatible perspectives to simultaneously have determinate value. Just as in quantum physics, the system (here our mental picture) makes discrete jumps when attempting (by means of introspection) to find a determinate value along a series of incompatible perspectives. ${ }^{2}$

\footnotetext{
${ }^{2}$ Let us take an example. we are interested in learning about a state agency. We may learn everything about the recruitment system which informs about the qualifications of people in various positions. It is a personnel perspective that we can map back to a perspective guided by say a concern about the correctness of a decision. If we next learn about the allocation of responsibility and associated path of decisions, it activates other concepts (another network of neurones). We then try to map back into a
} 


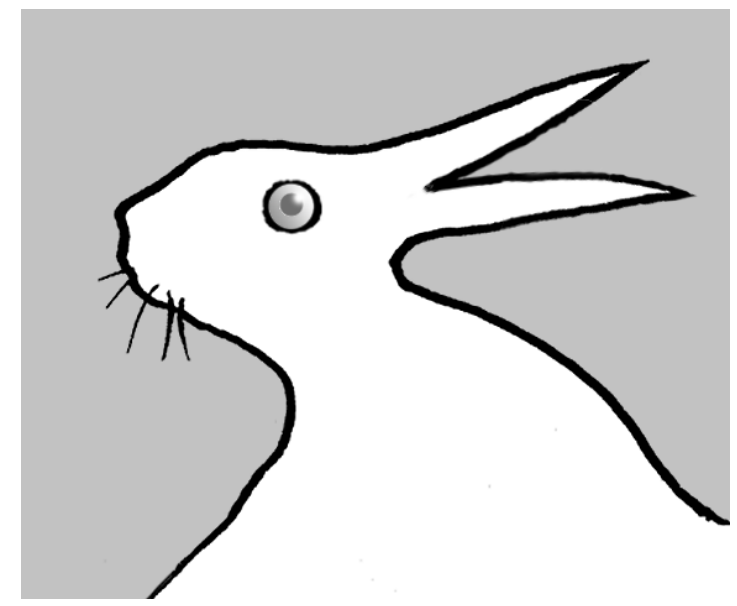

Figure 1. What do you see?

We model the cognitive process of learning as involving two essentially different operations. The first operation corresponds to receiving information framed according to some perspective. We model this as a preparation procedure. The second operation entails processing information or updating the mental picture in terms of one's preferred perspective. It is modeled as an introspective measurement operation. This learning process is not Bayesian as soon as we allow for Bohr complementarity of perspectives. The issue of learning by non-classically minded agents has been approached in Danilov and Lambert Mogiliansky [7, 8] and more recently in Busemeyer and Bruza [4].

\section{The basic model}

The parallel with QM is as follows: the system is the represented world, it is a psychic object. At any point in time the individual's knowledge about the (represented) world is captured by the current mental picture or state. We denote it $|\varphi\rangle \in \mathbb{H}$ where $\mathbb{H}$ is finite dimensional Hilbert space of mental pictures of the (relevant) world. The state can be characterized from different perspectives. A perspective is modelled as an operator similar to an observable. It operates on the mental picture to generate an outcome, a possible (set of ) picture(s) belonging to that perspective. The present analysis addresses incomplete knowledge due to either incomplete information or quantum indeterminacy or both.

\section{1-1. The notion of measurement}

Before entering into the analysis let us remind of some basic notions and how they relate to our issue. Generally, a measurement is an interaction between a system and some measurement device, which yields some result, the outcome of the measurement that we can observe and record. ${ }^{3}$ Two measurements are compatible if they, roughly speaking, can

perspective, we find out that it is not consistent with out earlier held beliefs.

${ }^{3}$ For instance in the case of the Stern-Gerlach experimental setup, we let the electron travel through a non-homogeneous magnetic field and observe deviation either up or down. In another example we let people play the Prisoner Dilemma (or the UG) and observe their choice. In the present context we see part of the cognitive process (see below) as a measurement of the mental picture. 


\section{ARIAne LAMBERT-Mogiliansky ANd François Dubois}

be performed simultaneously or more precisely, if the performance of one measurement does not affect the result of the other. Suppose that the first measurement gave outcome $o$; then we perform the second measurement and the first one anew. In case we are dealing with compatible measurements we obtain outcome $o$ with certainty. If all measurements are compatible we can substitute them with a single finest (complete) measurement, which is also first-kind. ${ }^{4}$ Performing that measurement we learn everything about the system. Such a system is classical. The existence of incompatible measurements is a distinctive feature of non-classical systems. It is closely related to the impact of measurements on the state and to the existence of "dispersed" states. A state is dispersion-free when the outcome of every possible measurement is uniquely determined, there is no reason for the state to change. If all pure states are dispersion-free then measurements do not impact on pure states and therefore all measurements are compatible. On the contrary, if a state is dispersed then by necessity it will be modified by an appropriate measurement. On the other hand, the change in a pure state is the reason for incompatibility of measurements. The initial outcome of a first measurement is not repeated because the system has been modified by a second measurement (see Danilov and Lambert-Mogiliansky [7, 8]).

In this paper a measurement is an introspective operation that acts on the agent's mental picture. The agent's asks himself a question: what are the implications of new information for my understanding of a specific matter? and an answer, the outcome of the mental process is brought to consciousness e.g., my current understanding in terms of a recommendation for action. We propose that some cognitive limitations documented by practitioners (give references) can be suitably modelled as the result of incompatible mental operations that act on the mental picture to modify it.

\section{1-2. $\quad$ Pure and mixed mental states}

A pure state is a maximal information state. It may be the eigenstate of some perspective so it gives maximal and precise knowledge of the world along some perspective. Or it may be a superposed state i.e., not the eigenstate of any perspective (observable). Such a state also contains maximal knowledge but is characterized by some "dispersion entropy" (see definition below). No measurement can be performed on a pure state and provide new information without losing some information. ${ }^{5}$ In contrast a mixed state is a familiar probabilistic combination of states which reflects incomplete knowledge. Measurements can be performed on a mixed state to reach a maximal information pure state. In this section we briefly discuss how a mixture of mental states can emerge.

At a first a mixed state seems to be a most natural mental object. So for instance the "world" is a friend's mood. You may be able to assign a probability to her willingness to go out with you. Although that situation appears to be most ordinary, we need to make

\footnotetext{
${ }^{4}$ If we can perform a measurement twice in a row. If the outcomes of the two measurements always coincide, we say that the measurement is a first-kind measurement.

${ }^{5}$ When the state already is a the eigenstate of the observable than no information is either lost or gained.
} 


\section{Our (Represented) World: A QuAntum-Like OBject}

clear how such a mixed state may arise in our context. In the world of QL-minded agents there exist three possible scenario:

- the state has been prepared to a mixed state i.e., information provided in terms of a probability distribution over possible states $^{6}$;

- an introspective complete measurement is initiated but not completed. The initial superposition has decohered into a probabilistic mixture of the possible states but not collapsed into one of the eigenstates; ${ }^{7}$

- a superposed state has spontaneously decohered;

In the present paper we shall not consider spontaneous decoherence as we do not explicitly consider time evolution. We focus on probabilistic mixture induced by the environment through well-identified new information or by means of an introspective mental processes.

\section{1-3. Intentionality: a preferred basis}

We shall assume that an individual endeavours to construct a representation i.e., learn about the world because he has some concern in mind e.g., he has to select an action. This assumption is consistent with psychological and neurobiological evidence that human cognitive processes (including perception) are structured by some form of intentionality.

A perspective is defined as an "observable" that is an operator $R$ that applies on the state or mental picture. It is characterized by its eigenvalues $\left\{r_{1}, \ldots r_{n}\right\}$ interpreted as the possible eigenpictures belonging perspective $R$. A perspective is a "complete set of commuting observables" so that a eigenstate in any perspective is a maximal information state. Consequently and importantly any two perspectives $R \neq P$ yield alternative descriptions of the same "world". A pure mental picture can written as a superposition of the possible eigenpictures in any perspective of the (relevant) world:

$$
|\varphi\rangle=\sum_{i} \lambda_{i}\left|r_{i}\right\rangle, \quad \sum_{j} \lambda_{i}^{2}=1, \lambda_{i} \in \mathbb{R}
$$

which can also writes as a density operator

$$
\rho=|\varphi\rangle\langle\varphi|
$$

When the initial mental picture is not a pure state but a statistical mixture of pure states, it can only be represented with a density operator $\rho$ :

$$
\rho=\sum_{i} \tau_{i}\left|\varphi_{i}\right\rangle\left\langle\varphi_{i}\right|, \quad \tau_{i} \geq 0, \quad \sum_{j} \tau_{i}=1
$$

where $\left|\varphi_{i}\right\rangle\left\langle\varphi_{i}\right|$ is a classical "ket-bra" notation to represent the orthogonal projector on the vector $\left|\varphi_{i}\right\rangle$. The density operator allows to express a situation where the agent's mental state is characterized by both intrinsic uncertainty and incomplete information.

\footnotetext{
${ }^{6}$ You know your friend received the results of a determinant exam but only the probability for the outcomes, if she passed she is in a mood to go out.

${ }^{7}$ You have reflected over her mood and formulated the uncertainty without "solving it" for yourself.
} 


\section{ARIAne LAMBERT-Mogiliansky ANd François Dubois}

An action problem is defined as a correspondence from $D: \mathbb{H} \rightarrow A$ where $\mathbb{H}$ is the space of mental pictures of the world and $A$ the set of actions. For each (mentally represented) state of the world, it defines which actions the agent wants to undertake.

\section{Hypothesis 1}

There exists a perspective $R^{*}$ such that $D$ is a coarsening of $R^{*}{ }^{8}$

Hypothesis 1 implies that if the agent receives (maximal) information framed in perspective $R^{*}$ he will know exactly what to do. We also say that $R^{*}$ is fully congruent with $D$. Generally, $D$ has a lower dimensionality than $\mathbb{H}$, i.e., distinct eigenpictures can call for the same action.

\section{1-4. The learning process}

In our context, it is in place to distinguish between two essentially different operations in the learning process ${ }^{9}$. We shall present them as two successive phases.

The first phase of the mental process is an interaction between the mind (mental picture) and the outside world. The individual acquires new information expressed within some perspective. This is a very important point: information is not "neutral", it is always framed i.e., it comes together with a perspective (model) of the world. Because it is framed, to integrate a piece of information, the individual may have to (temporarily) switch to the perspective in which it is formulated. Either the information belongs to the initial perspective $R$ or to some alternative perspective $P, P \neq R$. In both cases information acquisition corresponds to a fully deterministic evolution of the mental picture. It operates on the initial state(picture) so as to project it onto the one picture encoding the information content provided. In the terminology of QM, phase 1 resembles a process of preparation ${ }^{10}: \rho \rightarrow \rho^{\prime}$ where $\rho^{\prime}$ is the new density matrix. Let $\rho=\sum_{i} \theta_{i}\left|r_{i}\right\rangle\left\langle r_{i}\right|, \sum_{i} \theta_{i}=1$. If the information is consistent with $R, \rho^{\prime}=\sum_{i} \theta_{i}^{\prime}\left|r_{i}\right\rangle\left\langle r_{i}\right|$, where $\theta_{i}^{\prime}$ are calculated according to the familiar Bayes rule. We have a classical case of belief updating. The interesting case is when $P \neq R$, the mental picture is then "forced"11 into the perspective $P$ and $\rho /$ in basis $\left\{\left|p_{j}\right\rangle\right\}$ writes:

$$
\rho^{\prime}=\sum_{i} \gamma_{i}\left|p_{i}\right\rangle\left\langle p_{i}\right|, \sum \gamma_{i}=1
$$

\footnotetext{
${ }^{8} \mathrm{~A}$ measurement $M^{\prime}$ is coarser than $M$ if every eigenset of $M$ is contained in some eigenset of $M^{\prime}$, see Danilov and Lambert-Mogiliansky [7, 8] p. 334.

${ }^{9}$ Describing the two phases as essentially different is a simplification because information acquisition is not "direct" either. It also involves mental processes: perception is not neutral and not always classical (cf quantum zeno effect). Nevertheless a major distinction between the 2 phases is that the first involves an input from the outside while the second does not.

${ }^{10}$ In Physics a preparation procedure operates on a large number of systems to filter those whose properties are the desired ones. But one can also prepare a single system into a specific state by submitting it to a series of measuremnt until de desired result is obtained. The idea we retain is that the procedure secures that the system is in a specified state.

${ }^{11}$ Clearly the individual could neglect the information and he would not be forced into anything. But if he wants to integrate it, he must exert some mental flexibility and switch to the perspective in which that information takes its meaning.
} 


\section{Our (Represented) World: A QuAntum-Like OBject}

Depending on the provided information content the density matrix may be a pure state or mixed state (if the information content is probabilistic).

A first central point to emphasize is that when $P R \neq R P$ i.e., we are dealing with incompatible (alternative) perspectives, information embodied in $\rho$ the initial state is lost in the process. A preparation in $P$ modifies the mental picture into a specific mental picture distinct from the initial one and the result is the same whatever the initial state in $R$. Again this would not have been the case if the provided information was in terms of the same perspective as the initial state $R$. Of course this is a simplification but it it aims at capturing the following. The loss of information reflects the fact that the two pieces of informations $\left(\rho\right.$ and $\rho^{\prime}$ ) do not form a consistent picture for the individual. It is as if they are expressed in two different languages that do not translate into each other unambiguously and the individual cannot but think (form a mental picture) in either one. In reality, the process is of course much more complex. In particular, as earlier mentioned the individual may keep some memory.

Phase 2: Updating The second phase is a purely mental operation which does not appeal to the outside world. It is an introspective measurement operation. It measures the mental state $\rho^{\prime}$ with respect to the preferred perspective $R^{*}$. This operation may be conceptually decomposed into two steps in a way originally proposed by von Neuman (1932). The first step corresponds to the formulation of the mental picture in terms of a convex combination (probabilistic mixture) of possible outcomes in the preferred perspective $R^{*}$. This corresponds to making the measurement but not registering the result. The state is projected on the preferred basis and decohered. The second step entails a decision - one (or a subset of possible) result is singled out. It corresponds to registering the result of the measurement. We shall refer to these two steps as respectively analysis and decision.

\section{a. Analysis}

Generally, the concerns that determine intentionality may not require a complete measurement. So both the analytical step and the decision step may be coarse (as opposed to fine or complete measurement). In that case the outcome will entail the preservation of some quantum coherence so the state obtained after the decision is also a superposition. We next describe the process for a complete measurement which fully decoheres the state into a mixture of eigenpictures of $R^{*}$ before selecting one of them. The state $\rho^{\prime}$ is expressed in terms of the projectors associated with the eigenvectors of $R^{*}$ :

$$
\rho^{\prime}=\sum_{k, l}\left(\sum_{i} \gamma_{i}\left\langle r_{k}^{*}|| p_{i}\right\rangle\left\langle p_{i}|| r_{l}^{*}\right\rangle\right)\left|r_{k}^{*}\right\rangle\left\langle r_{l}^{*}\right|
$$

The $\rho^{\prime}$ contains off the diagonal elements which do not correspond to any eigenstate of $R^{*}$, they cannot be observed. The analysis step associated with a complete measurement corresponds to projecting the state into the $R^{*}$ perspective, i.e., decoherence takes place 
so only diagonal elements are left: $\rho^{\prime} \rightarrow \rho^{*}=\sum_{j}\left|r_{j}^{*}\right\rangle\left\langle r_{j}^{*}\left|\rho^{\prime}\right| r_{j}^{*}\right\rangle\left\langle r_{j}^{*}\right|$ and we write $\rho^{*}$ :

$$
\rho^{*}=\sum_{k} \theta_{k}^{*}\left|r_{k}^{*}\right\rangle\left\langle r_{k}^{*}\right|
$$

with diagonal coefficients $\theta_{j}^{*}=\sum_{i} \gamma_{i}\left|\left\langle p_{i} \mid r_{j}^{*}\right\rangle\right|^{2}, \sum_{j} \theta_{j}^{*}=1$. This new density operator is the result of "analysis" in the sense that the possible results have been identified with their respective probability for occurrence. Some information from the preparation stage is "transmitted" modulo the correlation coefficients $\left|\left\langle r_{i}^{*} \mid p_{j}\right\rangle\right|$ which are the probabilistic weights attached to the eigenstates in $\rho^{*}$. Some information is "lost" with the disappearance of the non-diagonal elements. The correlation coefficients linking two representations are features of the mind; they may but need not reflect features of the world that is represented. This is a fundamental distinction between quantum cognition and quantum mechanics. In Quantum Mechanics the correlation coefficients pertains to observables representing properties of a physical system. The subject matter of the present investigation is not the world but the represented world which is a mental construct. In particular, our model applies to the representation of classical objects. The coefficient captures the quantum-like structure of the mind actualized in cognitive activity.

\section{b. Decision}

The decision step is a non-deterministic process. When the updating phase corresponds to a complete measurement, the resulting density operator represents an eigenpicture of the preferred perspective ${ }^{12}$. Decision selects one of the $\left|r_{j}^{*}\right\rangle\left\langle r_{j}^{*}\right|$ with the corresponding probability $\theta_{j}^{*}$

$$
\rho^{*} \rightarrow \rho^{* *}=\left|r_{j}^{*}\right\rangle\left\langle r_{j}^{*}\right| .
$$

At this point, the individual has a maximally informed mental picture in $R^{*}$ and all information in $P$ from $\rho^{\prime}$ has been "lost". Recall that it affected the mental process through the probability for obtaining any of the possible results. We see that after completing the cognitive process corresponding to interpreting the information in his own mental model, the agent's actual state of information is a maximal information state which cannot be easily traced back to the initial $\rho$ or even to intermediary $\rho^{\prime}$ and $\rho^{*}$. We next develop a simple numerical example.

\section{Example}

In this section we illustrate the learning process defined above in numerical example. Consider a two dimensional Hilbert space and a 2X2 density matrix in the canonical preferred basis $R^{*}$. Let $\rho$ represent the initial state $\rho=.25\left|r_{1}^{*}\right\rangle\left\langle r_{1}^{*}|+0.75| r_{2}^{*}\right\rangle\left\langle r_{2}^{*}\right|$

$$
\rho=\left(\begin{array}{cc}
.25 & 0 \\
0 & .75
\end{array}\right)
$$

Phase 1: Information acquisition

\footnotetext{
${ }^{12}$ The state resulting from a coarse measurement is a pure state that is a superposition of eigenpictures.
} 
Consider an alternative perspective $P$ with two eigenpictures $\left\{p_{1}, p_{2}\right\}$. The basis $\left\{\left|p_{1}\right\rangle,\left|p_{2}\right\rangle\right\}$ corresponds to a rotation of $\left\{\left|r_{1}^{*}\right\rangle,\left|r_{2}^{*}\right\rangle\right\}$ by an angle of $\pi / 8$. The agent receives incomplete information in $P$ i.e., the true state is either $p_{1}$ or $p_{2}$ with respective probability $\gamma_{1}=(2+\sqrt{2}) / 4$ and $\gamma_{2}=(2-\sqrt{2}) / 4$. The density operator representing the state that obtains as a result of the acquisition of the new information is $\rho^{\prime}=(2+\sqrt{2}) / 4\left|p_{1}\right\rangle\left\langle p_{1}|+(2-\sqrt{2}) / 4| p_{2}\right\rangle\left\langle p_{2}\right|$. Note that $\rho^{\prime}$ does not depend on $\rho$, it only reflects the newly acquired information.

Phase 2: Updating

The density operator must now be written in terms of the $\left|r_{i}^{*}\right\rangle\left\langle r_{i}^{*}\right|$ projectors $\rho^{\prime}=$ $.75\left|r_{1}^{*}\right\rangle\left\langle r_{1}^{*}|+0.25| r_{2}^{*}\right\rangle\left\langle r_{2}^{*}|+0.25| r_{1}^{*}\right\rangle\left\langle r_{2}^{*}|+.25| r_{2}^{*}\right\rangle\left\langle r_{1}^{*}\right|$ or

$$
\rho^{\prime}=\left(\begin{array}{ll}
.75 & .25 \\
.25 & .25
\end{array}\right)
$$

After the analysis step, the matrix $\rho^{\prime}$ given in (2) becomes

$$
\rho^{\prime \prime}=\left(\begin{array}{cc}
.75 & 0 \\
0 & .25
\end{array}\right)
$$

Only the diagonal elements (representing observable states) are left. If we compare the matrices $\rho$ and $\rho^{\prime \prime}$, we observe that the probabilities associated with the eigenpictures of the preferred perspective have been inverted so if we complete the measurement, there is now $75 \%$ chance that the agent ends up being convinced of $r_{1}$ instead of $25 \%$ as before he acquired new information. In order to better understand why this is a reflection of cognitive constraints we need to introduce the concept of dispersion entropy and explore further the example which we do below.

\section{Learning under irreducible uncertainty}

\section{2-1. Dispersion entropy - a measure of doubt}

In this section we propose a concept of entropy that captures the amount of (relative) uncertainty that characterizes the mental picture of an agent. The first important point is that this uncertainty is not "absolute" but relative to a specific perspective e.g., corresponding to some objective that requires action. To make the point most clear we shall therefore confine ourselves to pure states i.e., states of maximal information. In those states, the corresponding mental picture is a superposition of eigenpictures i.e., the only uncertainty that is left is due to the quantum-like structure of the mind.

Shannon entropy which measures uncertainty associated with a classical probability is defined for a discrete probability distribution $\left\{p_{j}\right\}$ according to

$$
S=-\sum_{j} p_{j} \log p_{j}
$$




\section{Ariane Lambert-Mogiliansky and François Dubois}

It has been generalized by von Neumann to apply to quantum states and in particular to density operators. Let $\rho=\sum \lambda_{i}\left|\varphi_{i}\right\rangle\left\langle\varphi_{i}\right|$ :

$$
S(\rho)=-\operatorname{tr}(\rho \log \rho)
$$

or equivalently for

$$
S(\rho)=-\sum \lambda_{i} \log \lambda_{i}
$$

As does Shannon entropy, von Neumann entropy measures the amount of uncertainty due to incomplete information while we are interested in measuring intrinsic uncertainty. We therefore talk about the entropy of dispersion rather than of probability distribution. ${ }^{13}$ The relative entropy of dispersion is defined:

Definition The dispersion entropy of a pure state $\rho=|\psi\rangle\langle\psi|$ with $|\psi\rangle=\sum \lambda_{i}\left|\varphi_{i}\right\rangle, \sum \lambda_{i}^{2}=$ 1 relative to a specific perspective $A$ with eigenvectors $\left\{\left|a_{i}\right\rangle\right\}$ is

$$
S(\rho, A) \doteq-\sum \alpha_{j} \log \alpha_{j}
$$

where $\alpha_{j}=\sum_{i} \lambda_{i}^{2}\left|\left\langle a_{j} \mid \varphi_{i}\right\rangle\right|^{2}, \sum \alpha_{i}=1$.

A few important remarks:

1. Dispersion entropy is a relative concept, it varies with the perspective;

2. Dispersion entropy is equivalent with the von Neumann entropy of the mental picture $\rho^{\prime}=\sum \alpha_{i}\left|a_{i}\right\rangle$ decohered by the measurement of $A$ but before the decision (see analysis in Phase 2 of section 2.2 ).

3. $S(\rho, A)=0$ when the mental picture $|\varphi\rangle$ is an eigenpicture of $A$ and is positive otherwise.

In contrast with the standard concept of entropy we cannot say that the dispersion entropy of a pure state measures how much information we might gain by actually performing the measurement - because we are in a zero von Neumann entropy state. The $S(\rho, A)$ of pure state $\rho$ captures the non epistemic impact of the operation of measurement of $A$ on the mental picture ${ }^{14}$

In the context of quantum cognition we would like to link dispersion entropy to a psychological state of "doubt". ${ }^{15} S(\rho, A)$ is then a measure of the extent of "inner uncertainty". It can be resolved by an introspective measurement. ${ }^{16}$ An introspective measurement does not only take the logical consequence of information (the projection that decoheres the state), it also includes a resolution (what we called "decision"). ${ }^{17}$ Another

\footnotetext{
${ }^{13}$ See sect 2.2 above.

${ }^{14}$ Clearly the definition could be extended to include arbitrary state i.e., mixed state. It would then capture the combined uncertainty from incomplete information and intrinsic uncertainty and would remain a relative concept.

${ }^{15}$ The term doubt is used in it s psychological meaning i.e., not the cartesian rational doubt that defines the scientific approach.

${ }^{16}$ It could also be resolved by information acquisition in perspective $A$.

${ }^{17}$ Another way to put it is that an introspective measurement is a way to deal with "doubts" where doubt is not (only) a problem of information but an inner state of lack of conviction.
} 


\section{Our (Represented) World: A QuAntum-Like OBject}

way to put it is that a (complete) introspective measurement operates on a pure mental picture to resolve a state of doubt or lack of conviction.

We next develop a simple argument showing how additional information can increase dispersion entropy relative to the preferred basis. For the purpose of the argument, we assume that the agent's mental state is initially an eigenpicture of his preferred perspective $|\varphi\rangle=\left|r_{2}^{*}\right\rangle$. This state reflects information that is perfectly congruent with $R^{*}$ but is framed in $R:|\varphi\rangle=\sum \lambda_{i}\left|r_{i}\right\rangle\left(=\left|r_{2}^{*}\right\rangle\right)$. The agent is not aware of $r_{2}^{*}$ unless he updates "his beliefs" which corresponds to the (measurement) operation of $R^{*}$. If he did, he would obtain

$$
R^{*}|\varphi\rangle=\left|r_{2}^{*}\right\rangle
$$

with probability 1 . He would then be fully determined i.e., convinced with respect to the decision problem. There would not be any (relevant) uncertainty left with $\gamma_{2}=1$ we have $S\left(\rho, R^{*}\right)=0$. But assume that instead he acquires new information relative to another but related aspects of the issue. We denote this perspective $P$. This corresponds to steps 1 of the cognitive process above. The mental state $|\varphi\rangle$ is prepared into $\left|\varphi^{\prime}\right\rangle$ equal to some $\left|p_{i}\right\rangle$ i.e., information is maximal in perspective $P$. "Enriched" with this new information, the agent now updates his mental picture with respect to his preferred perspective (step 2):

$$
\left|\varphi^{\prime}\right\rangle=\left|p_{2}\right\rangle=\sum_{j} \gamma_{j}\left|r_{j}^{*}\right\rangle \rightarrow \text { any }\left|r_{j}^{*}\right\rangle \text { with probability } \gamma_{j}^{2} \neq 1
$$

We see that while he previously potentially "knew" $r_{2}^{*}$ (but was unaware of it) he is now in a state of hesitation where he believes that there is only a probability of $\gamma_{2}^{2}<1$ that the "true" eigenpicture is $r_{2}^{*}{ }^{18}$ The acquisition of new information has triggered the loss of "conviction" reflecting previously held information which is in effect lost leaving the agent in a state of intrinsic uncertainty captured by a strictly positive dispersion entropy:

$$
S\left(\rho^{\prime}, R^{*}\right)=-\sum_{j} \gamma_{j}^{2} \log \gamma_{j}^{2}>0
$$

where $\rho^{\prime}=\sum_{j} \gamma_{j}^{2}\left|r_{j}^{*}\right\rangle\left\langle r_{j}^{*}\right|$.

Proposition. Assume that the agent's mental state is one eigenpicture of his preferred perspective, acquiring additional information in terms of a perspective that is not compatible with the preferred one affects the mental state so as to increase dispersion entropy reflecting doubt or lost conviction.

This is an important result. From the point of view of "inner conviction" additional information is unambiguously detrimental when the starting point is an eigenpicture of the preferred perspective as illustrated in the argument above: the new information necessarily induces uncertainty with respect to the agent's action relevant perspective. More generally i.e., starting from an arbitrary eigenpicture (possibly mixed and with non zero relative dispersion entropy) as in the example above, new information may either decrease

\footnotetext{
${ }^{18}$ After the introspective process, he will end up believing $r_{2}^{*}$ with some probability less than 1 .
} 


\section{ARIAne LAMBERT-Mogiliansky ANd François Dubois}

or increase dispersion entropy. In the next section we discuss possible implications for rational learning.

Remark 1 In our case $r_{2}^{*}$ is true because it reflects an information congruent with $R^{*}$ and $\left|p_{i}\right\rangle$ is also true. The agent is not mistaken. He simply cannot hold in one single picture both informations. The introspective operation when processing information $p_{2}$ involves the whole (cognitively limited) mind and therefore upsets earlier held beliefs..

We illustrate the result in Proposition 1 in figure 2 where the dimensionality of a perspective is 2 . The broad line corresponds to the preparation stage and the thin lines to the measurement.

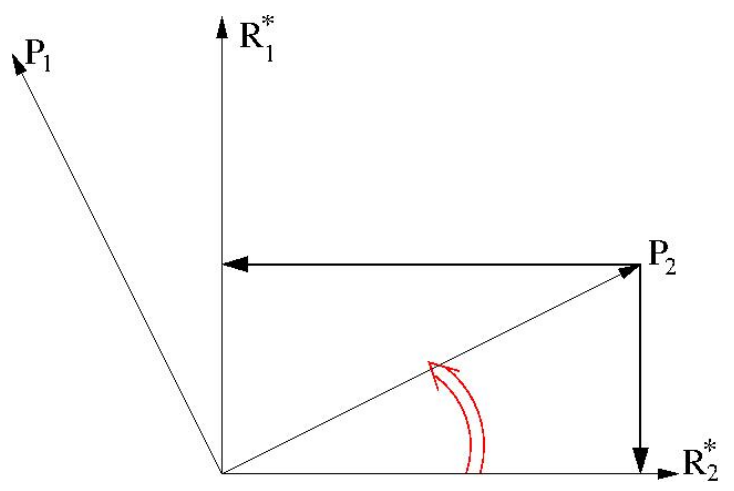

Figure 2. Detrimental information

\section{Example cont.}

Using the numerical example above, we illustrate the impact of new information on dispersion entropy. The starkest results appears when starting out with $\rho=\left|r_{2}^{*}\right\rangle\left\langle r_{2}^{*}\right|$ and assume that the state was generated as the result of the acquisition of maximal information in perspective $R^{*}$ and thus associated with zero dispersion entropy relative to $R^{*}$. After acquiring information the state of knowledge/conviction is $\rho^{\prime}=\left|p_{1}\right\rangle\left\langle p_{1}\left|=\frac{2+\sqrt{2}}{4}\right| r_{1}^{*}\right\rangle\left\langle r_{1}^{*}\right|+$ $\frac{\sqrt{2}}{4}\left(\left|r_{1}^{*}\right\rangle\left\langle r_{2}^{*}|+| r_{2}^{*}\right\rangle\left\langle r_{1}^{*}\right|\right)+\frac{2-\sqrt{2}}{4}\left|r_{2}^{*}\right\rangle\left\langle r_{2}^{*}\right|$. Then $\rho^{\prime \prime}=\frac{2+\sqrt{2}}{4}\left|r_{1}^{*}\right\rangle\left\langle r_{1}^{*}\left|+\frac{2-\sqrt{2}}{4}\right| r_{2}^{*}\right\rangle\left\langle r_{2}^{*}\right|$. The new state is associated with the following dispersion entropy $S\left(\rho^{\prime}, R^{*}\right)=-\frac{2+\sqrt{2}}{4} \log \left(\frac{2+\sqrt{2}}{4}\right)-$ $\frac{2-\sqrt{2}}{4} \log \left(\frac{2-\sqrt{2}}{4}\right)=0.4165>S\left(\rho, R^{*}\right)=0$.

So now we readily see that new information created a state of doubt when there should not have been any - the agent has maximal information with respect to the perspective relevant to his decision. The doubt does not arise as the result of the information content associated with $\left|p_{1}\right\rangle\left\langle p_{1}\right|$ but exclusively as the result on the cognitive constraint expressed by the incompatibility between the two perspectives corresponding to $R^{*}$ and $P$ respectively.

Remark 2 It must be emphasized if we relax slightly the assumption that $|\varphi\rangle=\left|r_{2}^{*}\right\rangle$ (i.e., allowing for initial incomplete information), the result in the Proposition can be obtained with a classical model of a different flavor. ${ }^{19}$ Assume the agent has priors that puts nearly all the weight (but not all) on $r_{2}^{*}$. Next, he receives information $p^{2}$. If

\footnotetext{
${ }^{19}$ See Dubois and Lambert-Mogiliansky (2014)for a detailed argument.
} 


\section{Our (Represented) World: A QuAntum-Like OBject}

according to the agent's structural model, that information is highly correlated with $r_{1}^{*}$, he updates his beliefs and is now less certain about what to do than he was before he got the information. His new beliefs exhibit a distribution with higher entropy. However, he has not lost information as in the quantum case. Instead the interpretation is that his priors were wrong so new information brought him closer to the truth.

In view of the remark above a legitimate question is: are we only talking about interpretation or do quantum cognitive limitations really have a more profound bearing on learning? The next section addresses this question.

\section{2-2. Rational Learning under Irreducible Uncertainty}

Learning whether it is by classically minded agent or QL-minded ones is always about the construction of a mental representation of the world. In our context it is important to distinguish between two properties of a representation. On the one hand the mental picture may be more or less informationally complete. And on the other hand, the mental picture may be more or less connected with the outside world it is supposed to represent. In a classical context this distinction also exists and information from the outside world is used to complement and test the mental representation. If the picture is incomplete, new information will bring it closer to the outside truth. If the model is wrong attempts to update it with new information will give rise to inconsistencies. It should be stressed that no satisfactory approach exists as to how a classically minded agent proceeds in face of data revealing failures of the underlying model. There is no obvious incremental path of modifications (the notion of distance is ambiguous at best) and certainly no prescription for discrete jumps between alternative models.

For a quantum-like agent, the situation is similar except that there is not one single "true" model (mental picture) but a multiplicity of Bohr complementary (correlated) "true" models. Discrete jumps between representations are parts of the cognitive process by force of complementarity. With quantum cognition the link between the epistemic state (the mental picture) of the represented world and the world in itself is ambiguous. In fact the term "epistemic state" does not seem proper in face of intrinsic indeterminacy as the multiplicity of zero entropy states suggests. As repeatedly emphasized by cognitive scientists we cannot address knowledge without simultaneously addressing human cognitive activity. As we shall see this takes a precise meaning in our context.

\section{2-2-1. Bayesian and QL-learning}

Bayesian learning operates within a Boolean algebra. The objective is to learn the parameters of the model of the system. ${ }^{20}$ Provided the priors are not inconsistent with the true model, we know (Schwartz 1965) that starting from any such priors Bayesian updating converges to the true state.

With quantum learning, we are in a Hilbert space, that is there exists a variety of resolutions of the system i.e., a variety of perspectives, of valid theories of the system.

\footnotetext{
${ }^{20}$ Some consider also Bayesain updating with multiple priors (see e.g., Hanany and Klibanov [18]). But there is no consensus as to how to proceed - in sharp contrast with Bayesian updating of single priors.
} 


\section{ARIAne LAMBERT-Mogiliansky ANd François Dubois}

Therefore, the state of knowledge of a quantum minded individual does not converge with new information but can evolve forever. Consider again the example above. After the agent performed the introspective measurement $R^{*}$, if the agent is being asked about his understanding in $P$, he will perform a new measurement of the eigenpicture resulting from the first measurement and $\rho^{\prime}$ will not be recovered. Performing those measurements alternately, he will keep oscillating without converging i.e., without being able to settle for a definite value in both $P$ and $R^{*}$ simultaneously. ${ }^{21}$ Of course this example is simplistic and the agent might simply remember $\rho^{\prime}$. But in more sophisticated context, we expect the modification of the mental picture to be effective.

In Quantum Mechanics, irreducible uncertainty gave rise to a rich philosophical debate about the nature of reality (see e.g., D'Espaganat 1979). In our context when the irreducible uncertainty is a consequence of the quantum structure of the mind, new questions arise. The mental picture can also "drift away" from a "truthful representation" of the outside world. ${ }^{22}$ We next briefly discuss what rational learning may mean in such a situation.

\section{2-2-2. The object of QL-learning and "Knowledge about the world"}

In quantum mechanics when physicists were confronted with the impossibility of first hand objectification, they resorted to second hand objectification. That is basically the Hilbert space model of quantum mechanics. It captures how measurements impact on the system and how measurements are related among each other. The state vector is the central object that encapsulates all information and it is the basis for making predictions. Similarly, the situation we are facing in quantum cognition suggests that the primary object of scientific learning must be the human mind i.e., the structure of mental perspectives, how they affect the mental representation of the world and how they are related to each other. The current mental picture is the state of the representation, it encapsulates the information known to the individuals and it is the basis for making prediction and further operations. This structure is assumed to exhibit invariance reflecting psychological regularities. ${ }^{23}$

Hence we propose that the primary object of QL-learning is the human cognitive process - the non-boolean algebra of mental perspectives. What knowledge about the outside world concern, we have seen that for QL-minded agents knowledge about the world is a mental picture that reflects both inputs from the outside world (information) and input from the inside world (decision in introspective measurement). At any point

\footnotetext{
${ }^{21}$ The general result is a transposition into cognition of the basic feature of quantum mechanics namely that it is not possible for complementary properties to have a determinate value simultaneously.

${ }^{22}$ The role of the acquisition of new information from an incompatible perspective is neither a complement nore a test of the truthfulness of the current picture. But it is an operation that forces a (temporary) reconnection between the mental picture and the outside world. Only information within the perspective of the mental picture is a true test in a way similar to learning in the classical world with classically minded agents.

${ }^{23}$ At least we do expect invariance at the individual level.
} 


\section{Our (Represented) World: A QuAntum-Like OBject}

of time the representation we have of the world depends on the path of information acquisition and introspective measurements performed. By choosing a specific path, the individual chooses - let be in a non-deterministic manner - his "world". He is the creator of the world he lives in. We know from psychologists that people systematically underscore their responsibility in failure (relative to bad luck) and overscore their impact on success. It has been suggested that this can be fully rational to optimize motivation and selfimage. ${ }^{24}$ The quantum cognitive approach provides a framework for effectively acting in this manner, it internalizes the (emotional) constraints that justify lying to oneself into cognition itself. Since QL-agents do have some freedom in the determination of the world they live in - it only seems rational to create a world in which we are more productive and feel good.

Rational learning about the world transforms into a choice problem the outcome of which affects our well-being in a way similar to other choices. As all creative processes, it is constrained - the cognitive process is structured. Moreover one should not end up with a mental picture that is fully disconnected with the outside world because that undermines the value of our actions which are based on that mental picture. But we are invited to recognize a significant freedom. We thus propose that rational construction of a representation of the world entails selecting what information to acquire and which introspective measurements to perform so as to produce a mental picture that maximizes our well-being in the actual world. For instance when having very specific practical concerns adequacy between the mental picture and the outside world may be particularly valuable. In such a case a strong conviction in a less relevant perspective obtained after a series of introspective measurements may benefit from being upset by new information. Future research is called upon to characterize rational learning by QL-agent in terms of optimal decision-making with respect to information acquisition and introspective measurements.

\section{Concluding remarks}

This paper is a first exploration of learning by cognitively limited agents where the limitations are modeled appealing to quantum like characteristics of the mind. A main motivation is that the quantum approach has shown successful in explaining behavioral anomalies in decision-making while it also seems able to capture concerns expressed by practitioners dealing with information transmission and communication. In particular, the fact that i. people reason about reality within the frame of some perspective ("mental script") or as we call it, a mental picture, ii, the multiplicity of potential representations of reality generates specific problems for learning. We model cognitive limitations in terms of the multiplicity of Bohr complementary mental representations of one and the same reality. We find that with intrinsic uncertainty, additional information induce loss of information that can be detrimental.

Our analysis calls for a new approach to learning. First, it puts the cognitive process

\footnotetext{
${ }^{24}$ The idea is that motivation is not fully maleable and must be incited: if you feel bad about yourself youmay not find the energy to make bold moves.
} 


\section{Ariane Lambert-Mogiliansky and François Dubois}

at the center: that is the logical structure behind the geometry of perspectives. Second, it unveils a form of creative freedom in the construction of a mental representation that invites a fully different approach. Namely learning about the world becomes a part of decision-making whose objective is to maximize well-being.

\section{References}

[1] Bitbol, M. (2009), Physique Quantique et Sciences Humaines, Edition CNRS: Paris, France.

[2] Bohm D. (1951), Quantum Theory, Prentice Hall, Englewood Cliffs.

[3] Bohr N. (1934), Atomic Theory and the Description of Nature, Cambridge University Press, London.

[4] Busemeyer J.R. and P. Bruza (2012), Quantum Models of Cognition and Decision, Cambridge University Press.

[5] Busemeyer J.R., Z. Wang and J.T. Townsend (2006), "Quantum Dynamics of Human Decision-Making", Journal of Mathematical Psychology, 50, p. 220-241.

[6] Busemeyer, J. R., E. Weg, R. Barkan, X. Li and Z. Ma (2000), "Dynamic and consequential consistency of choices between paths of decision trees", Journal of Experimental Psychology: General, 129, p. 530-545.

[7] Danilov V. I. and A. Lambert-Mogiliansky (2008), "Measurable Systems and Behavioral Sciences". Mathematical Social Sciences 55, p. 315-340.

[8] Danilov V. I. and A. Lambert-Mogiliansky (2008), "Decision-making under nonclassical uncertainty", in Proceedings of the the second interaction symposium (QI 2008), p. 83-87.

[9] Damasio A. (1995), L'Erreur de Descartes, Odile Jacob, Paris.

[10] Danilov V. I. and A. Lambert-Mogiliansky (2010), "Expected Utility under Nonclassical Uncertainty", Theory and Decision 2010/68, p. 25-47.

[11] Dawes S. (2010) "Stewardship and Usefulness: Policy principles for Information-based Transparency", Government Information Quartely, 27/4, p. 377-383.

[12] d'Espagnat B. (1979), À la recherche du réel - Le regard d'un physicien, GauthierVillars, Paris.

[13] Epstein L, J. Noor and A. Sandroni (2008), "Non-Bayesian updating: A theoretical framework", Theoretical Economics 3, p. 193-229.

[14] Gioia D. (1986) "Symbols, Script and Sense-making: creating meaning in the organizational experience", in Thinking Organization H. Sims and Jr D. Gioia and Associates (eds) Jossey Bass San-Fransisco, p. 49-74. 


\section{Our (Represented) World: A QuAntum-Like OBject}

[15] Heisenberg W. Philosophie: Le Manuscript de 1942, Edition Seuil: Heisenberg, Germany, 1998.

[16] Gilboa I. and D. Schmeidler (1989), "Maxmin Utility with non-unique priors", Journal of Mathematical Economics, p. 141-153.

[17] Gilboa I, A. Postlewaite and D. Schmeidler (2012), "Rationality of Beleifs or: why savage's axioms are neither necessary nore sufficient for rationality", Synthese, 187, p. 11-31.

[18] Hanany E. and P. Klibanov (2007), "Undating prefrences with multiple priors", Theoretical Economics, p. 261-298.

[19] Kahneman D. and A. Tversky (Eds.) (2000), Choices, values and frames, Cambridge University Press, New York.

[20] Khrennikov A. (2010), Ubiquitous Quantum Structure - From Psychology to Finance, Springer, New York.

[21] Lambert-Mogiliansky A., J.R. Busemeyer (2012), "Quantum indeterminacy in Dynamic decision-making: Self-control through identity management", Games, vol. 3/2, p. $97-118$.

[22] Lambert-Mogiliansky A., S. Zamir and H. Zwirn (2009), "Type indeterminacy - A Model of the KT(Khaneman Tversky)- man" Journal of Mathematical Psychology, vol 53/5, p. 349-361.

[23] Lambert-Mogiliansky A., F. Dubois (2015), "Transparency in public life ; a quantum cognition perspective", Proceedings of the QI-2014 conference, Lecture Notes in Computer Science, number 8951, p. 210-222, Springer.

[24] La Mura P. (2009), "Projective Expected Utility" Journal of Mathematical Psychology, p. 408-414.

[25] Noveck B. (2009), "Wiki Government: How Technology Can Make Government Better, Democracy Stronger, and Citizens More Powerful", Brookings Institution Press, Washington DC.

[26] Orlikowsky W.J. and D.C. Gash (1994), "Technological frames: making sense of information technology in organizations", ACM Transactions on Information Systems (TOIS), vol 12/2, p. 174-207.

[27] Pylkkanen Paavo (2014), "Can Analogies Helps Us to Understand the Process of Thought?" Mind $\&$ Matter 12/1, 61-91.

[28] Savage L. (1954), The Foundation of Statistics, Dover publication inc, New York.

[29] Zurech W. (2002), "Decoherence and the Transition from Quantum to Classical Revisited", Los Alamos Sciences November 27, 2002. 